\title{
Cervicogenic headache treated by acupuncture based on jin theory: study protocol for a randomized controlled trial
}

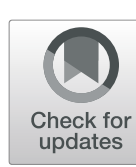

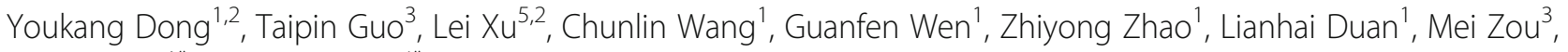
Yong Xiang ${ }^{1 *}$ and Shu Wang ${ }^{4^{*}}$ (D)

\begin{abstract}
Background: Numerous randomized trials involving acupuncture treatment for cervicogenic headache (CEH) have been conducted in recent years, but the evidence for its effectiveness is not clear. Hence, we designed a randomized trial to evaluate the efficacy and advantages of acupuncture for treating CEH.

Design: This is a parallel-design, two-arm, patient-assessor blinded, randomized, sham-controlled clinical trial. A total of 166 patients with CEH aged from 18 to 70 will be recruited and assigned randomly into a jin acupuncture group and a pseudo acupuncture group at a 1:1 ratio; they will receive 12 sessions of real acupuncture and sham acupuncture for 4 weeks, respectively, during the study. The primary outcomes are pain degree (PD) and pain rate (PR) calculated by the PainVision analyzer, as well as parameters detected by surface electromyography (SEMG). The secondary outcomes will be measured with the short-form McGill Pain Questionnaire (SF-MPQ), range of motion (ROM) of the neck, the Northwick Park Neck Pain Questionnaire (NPQ), the 36-item short-form Health Survey (SF-36), the Self-Rating Anxiety Scale (SAS), and the Self-Rating Depression Scale (SDS). Clinical assessments will be evaluated at baseline and in the fourth week as well as in the eighth and sixteenth weeks. Adverse events will be noted and recorded for the safety evaluation.
\end{abstract}

Discussion: This study will provide high-quality evidence of the value of acupuncture based on jin theory for treating $\mathrm{CEH}$.

Trial registration: Chinese Clinical Trial Registry, ChiCTR1800015316. Registered on 22 March 2018. Updated version AMCTR-IOR-18000157. Registered on 1 April 2018.

Keywords: Acupuncture, Cervicogenic headache, Randomized controlled trial, Study protocol

\section{Background}

Cervicogenic headache $(\mathrm{CEH})$ refers to a group of syndromes characterized by unilateral preponderance of headache, restricted movement of the neck, and hypersensitivity of the occipital neck region caused by degeneration of the cervical vertebra and detriment of the cervical soft tissue [1]. An epidemiological investigation showed that approximately $2.5 \%$ of individuals suffer

\footnotetext{
*Correspondence: 630458355@qq.com; 408750926@qq.com

${ }^{1}$ The First Affiliated Hospital of Yunnan University of Chinese Medicine (Yunnan Provincial hospital of Traditional Chinese Medicine), Kunming 650021, China

${ }^{4}$ The First Affiliated Hospital of Tianjin University of Traditional Chinese Medicine, Tianjin 300193, China

Full list of author information is available at the end of the article
}

from $\mathrm{CEH}$, with an age distribution between 30 and 50 years and a ratio of men to women of 1:4 [2]. The International Headache Society (IHS) considers CEH as a special disorder different from common types of headache [3], with published and revised reports in 2004 and 2013, respectively $[4,5]$. Although the pathological mechanism of $\mathrm{CEH}$ is still disputed, with causes focused mainly on musculoskeletal disorders in the cervical spine [6] or convergence afferent signal disturbance of the trigeminal nerve and the upper cervical nerves [7], there are various interventions being performed according to the accumulated experience, varying knowledge scopes, and professional trends of doctors, such as drugs (including narcotic drugs) [8], nerve block [9], pulse radiofrequency [10],

(C) The Author(s). 2019 Open Access This article is distributed under the terms of the Creative Commons Attribution 4.0 International License (http://creativecommons.org/licenses/by/4.0/), which permits unrestricted use, distribution, and 
Western medicine [11], acupuncture [12], Tuina [13], and Chinese medicine [14].

As a vital constituent of traditional Chinese medicine (TCM), acupuncture is well known and used in some countries. It has played a very important role in dealing with diseases associated with cervical spondylosis in China [15]. Some clinical trials demonstrated acupuncture to be superior to conventional methods regarding curative effects and unexpected events in the treatment of CEH [16, 17]. Jin theory, as an important branch of meridian theory, was demonstrated to be effective in the treatment of pain [18]. Clinically, according to TCM, jin disease basically appears as aches, clonuses, spasms, rigidity, relaxation, and limb weakness, symptoms which are very similar to the manifestation of muscle, tendon, myofascial membrane, ligament, and neuropathic lesions. Therefore, from the perspective of functional anatomy, jin refers to a complex system combining local anatomical morphology and significant characteristics mainly involving muscles, tendons, myofascial membranes, ligaments, and nerves, etc. [19]. CEH is a kind of jin disease, and its pathogenesis is closely related to the pain point of jin formed by pathological changes in muscles (cervical extensor muscles, myofascial fascia, and tendons) and nerves (occipitalis major nerve and occipitalis minor nerve) and abnormality of proprioception $[19,20]$. The aponeurosis attaches to the articular surface or vertebrae and becomes a stress concentration point due to the great stress caused by keeping the head and neck in a low flexion position. Long-term stress concentration can lead to the occurrence of energy metabolism crises of the muscle fibers, fibrosis of muscles, and other lesions, which contribute to form the tension zones and sensitive points of muscle fibers (gather and knot in TCM), inducing pain and distal referred pain when pressed [21]. In addition, when tension and spasm occur in the muscle where the nerve passes through, compression or stimulation symptoms are prone to be produced at the outlet of the nerve [22]. Therefore, taking the outlet of the nerve as an acupoint to perform acupuncture should show positive significance for the relief of symptoms of CEH.

At present, more attention is paid to the evaluation of headache, ignoring symptoms and signs of the neck and occipital region. Also, abundant non-acknowledged therapies have been adopted conventionally in control groups, which has weakened the clinical study quality of acupuncture for $\mathrm{CEH}$. In this study, we focus on the main and secondary symptoms and signs involving headache as well as pain and stiffness of neck-occipital region, and we aim to verify the value of acupuncture based on jin theory. The study and its final conclusion will provide a reasonable and high-quality clinical trial contributing to the popularization and application of acupuncture for $\mathrm{CEH}$.

\section{Methods and design Study design}

This is a two-arm, parallel-design, patient-assessor blinded, clinical randomized controlled trial (RCT) aiming to evaluate the differences of efficacy between acupuncture based on jin theory and sham acupuncture for CEH. A total of 166 patients will be recruited from the Department of Acupuncture-Tuina and Rehabilitation of the first affiliated hospital of Yunnan University of Traditional Chinese Medicine. Recruitment advertisements will be placed on the network and recruitment posters. Eligible participants in each group will have an equal chance of being allocated randomly to obtain real or sham acupuncture treatment. The primary outcomes are objective parameters detected by devices (PainVision analyzer and surface electromyography [SEMG]); the secondary outcomes are assessed by scales. This study will last 16 weeks including 1 week for baseline, 4 weeks for treatment, and 12 weeks for follow-up. Methods and data from this study will contribute to the feasibility and simplicity of acupuncture for $\mathrm{CEH}$ patients. This protocol was registered on 22 March 2018 (ChiCTR1800015316, AMCTR-IOR-18000157). The updated protocol was changed on 26 April 2019. Figure 1 shows a flowchart of the study. Additional file 1 provides the Standard Protocol Items: Recommendations for Interventional Trials (SPIRIT) checklist; Additional file 2 shows the Standards for Reporting Interventions in Controlled Trials of Acupuncture (STRICTA) checklist.

\section{Participants}

\section{Study population and sample size}

Patients with $\mathrm{CEH}$ meeting the diagnosis of the International Classification of Headache Disorders, 3rd edition (ICHD-3) formulated by the IHS [5] will be enrolled. The reduction of the visual analog scale (VAS) average score was 6.37 to 2.31 after triple acupuncture treatment compared to 6.37 to 2.52 for conventional acupuncture treatment according to a relevant study [23]. We propose to set the VAS of the short-form McGill Pain Questionnaire (SF-MPQ) average score as 2 and 4 for the jin acupuncture group and pseudo acupuncture group, respectively. Given a significance level of 0.05 , a standard deviation of 5 , and power of test of 0.95 , and considering a $10 \%$ loss rate, 166 participants in total are needed with 83 in each group.

\section{Inclusion criteria}

Volunteers who meet the following criteria modified from the Headache Classification Subcommittee of the IHS will be recruited: (1) headache originating from the neck and perceived in one or more regions of the head and/or face; (2) clinical, laboratory, and/or imaging evidence of a disorder or lesion within the cervical spine or soft tissue of the neck known to be a valid cause of headache; (3) 


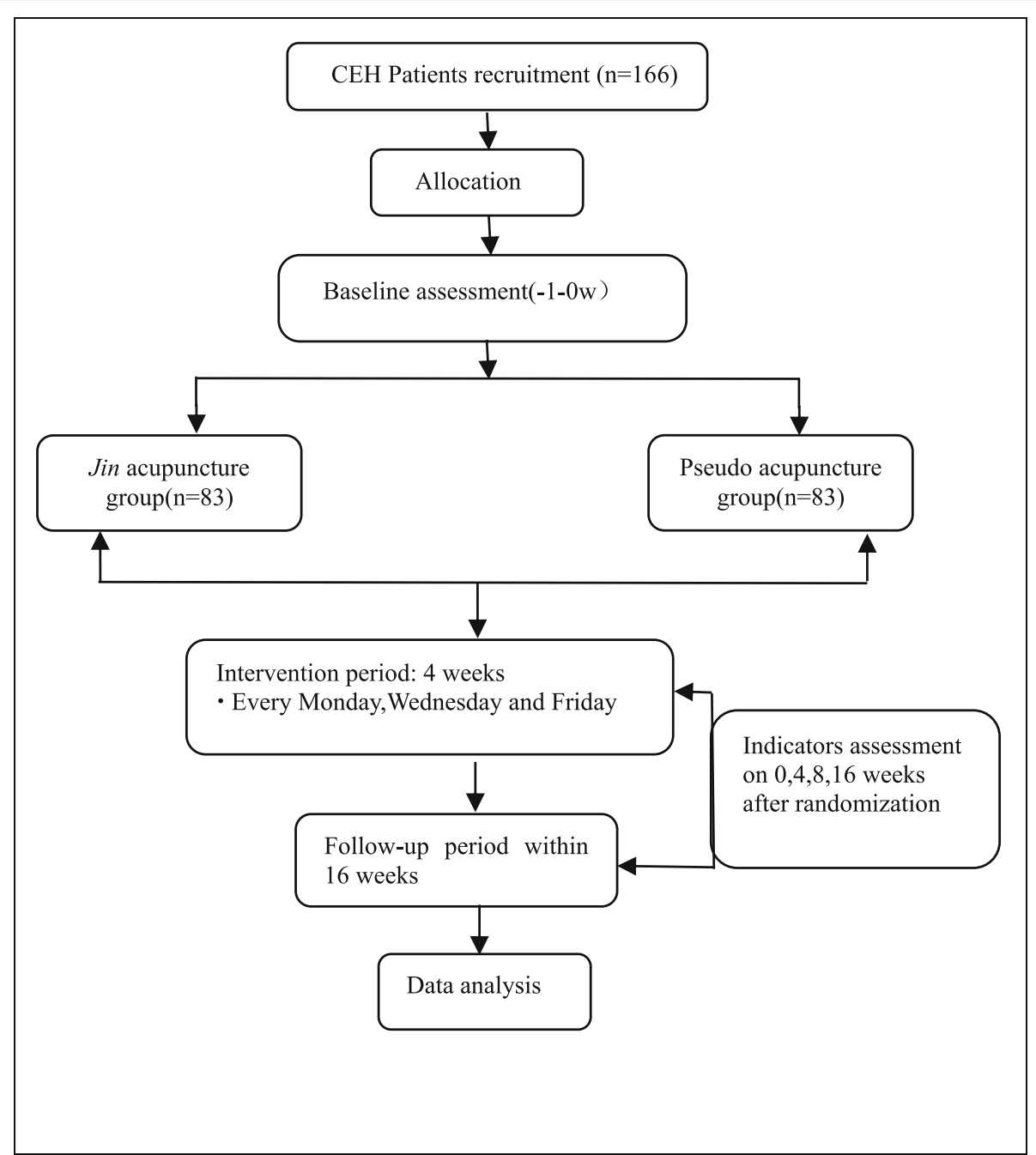

Fig. 1 Flowchart of this trial

clinical signs implicate a source of pain in the neck, and the headache can be attributed to the neck disorder or lesion; (4) aged from 18 to 70 years, both males and females; (5) have not received any treatment within 2 weeks; (6) willing to abide by and sign informed consent.

\section{Exclusion criteria}

Volunteers who meet any of the following criteria will be excluded: (1) headache related to tumor, cervical vertebral tuberculosis, spinal canal occupying lesions, or scoliosis or resulting from intracranial infection, cerebroma, subarachnoid hemorrhage, or other diseases; (2) have had operation for cervicogenic headache; (3) serious underlying diseases of important organs such as heart, liver, kidney, brain, or blood vessels, hematopoietic disorders or diabetes; (4) pregnant or lactating women; (5) unable to understand or record scoring indicators; (6) serious mental disorder, anxiety, or depression; (7) have received other treatment or involved in other clinical trials.

\section{Dropout criteria}

Patients who are unable to comply with this study and fail to achieve the expected efficacy, or who experience severe changes in their condition during treatment, will be dropped from the study.

\section{Randomization, allocation concealment, and blinding}

The eligible patients who meet the criteria will be randomly distributed to the jin acupuncture group and the pseudo acupuncture group with an allocation proportion of 1:1. A statistician who is not taking part in the clinical intervention will use SPSS 25.0 (IBM, Chicago, IL, USA) to generate a random allocation sequence. All of the random code information will be stored in sealed opaque envelopes by a well-trained specified assistant, who will randomize and inform the acupuncturists of the treatment assignments by phone. The allocation concealment procedure will not be exposed until the clinical trial is finished completely. Assessments and measurements of the participants will be 
carried out before and after the treatments and also at the follow-ups by different individuals of the research team. Researcher A will be responsible for the baseline assessment, administration of the questionnaire, and the measurement of cervical range of motion. Researcher B will be in charge of detecting the SEMG signal on the cervical extensor muscles. Researcher $\mathrm{C}$ will detect and record the pain degree (PD) and pain rate (PR) with the PainVision analyzer. Researcher D will be responsible for the analysis of SEMG data. A licensed acupuncturist who has worked for more than 18 years will perform acupuncture interventions on the patients, and patients will be treated in a shielded clinic room and deprived from knowing something about the pattern of treatment.

\section{Interventions}

Patients in the jin acupuncture group will receive 12 sessions of acupuncture during a period of 4 weeks, implemented by finding the gather and knot of positive reaction, the points of both stress concentration and neurogenic stimulation in cervical extensor muscles according to TCM jin theory and modern biomechanical principles of soft tissue. Sterile and disposable filiform needles (Suzhou Acupuncture \& Moxibustion Appliance Co. Ltd. Jiangsu, China) $40 \mathrm{~mm}$ in length and $0.30 \mathrm{~mm}$ in diameter will be inserted in accordance with the permissible depth and angle of the affected side acupoints until the de $q i$ sensation is achieved. Meanwhile, acupuncture techniques of lifting-thrusting and twirling-rotating will be implemented to facilitate the arrival of $q i$ involving a sensation of aching, numbness, heaviness, or distension around the acupoints. The needles will be retained for 25 min and stimulated every 8 min intermittently, with each acupoint being stimulated for $10 \mathrm{~s}$. Participants in the pseudo acupuncture group will receive the sham acupuncture using filiform needles on points $5 \mathrm{~cm}$ apart away from the acupoints used in the jin acupuncture group. The skin will be punctured without any manipulation and stimulation for 25 min to avoid the de qi sensation, with the same sessions and courses as the jin acupuncture group. The baseline period is 0 to week 1 with one evaluation time before participating. The course of treatment and observation period will be three sessions a week totaling 12 sessions during the course. After 4 weeks of treatment, we will provide one evaluation session. The follow-up evaluation will be performed in the eighth and sixteenth weeks. The study schedule is shown in Fig. 2; the treatment details for each group are listed in Table 1.

\section{Outcome measures}

\section{Primary outcome measures}

The following primary outcome measures will be used to evaluate the effectiveness at weeks 4,8 , and 16 after randomization, respectively, compared with the baseline.
(1) Pain degree (PD) and pain rate (PR): With a measurement accuracy of $0.1 \mu \mathrm{A}, 0.3 \mathrm{~ms}$ pulse width, and $50 \mathrm{~Hz}$ pulse frequency, both indicators will be calculated using the current perception threshold (CPT) and pain equivalent current (PEC) with the PainVision analyzer according to the following formula $[24,25]$ :

$$
P D=\frac{P E C-C P T}{C P T} \times 100 P R=\frac{P E C}{C P T}
$$

(2) Surface electromyography (SEMG): With the patient in a prone position, a sticker will be placed on the affected side cervical extensor muscles $(2 \mathrm{~cm}$ lateral to the posterior midline of the neck). The SEMG signal will be detected through an eightchannel module ME6000 biomonitor (Mega Electronics Ltd., Kuopio, Finland) as the operator successively applies $25 \%$ (maximum voluntary contraction, $\mathrm{MCV}$ ), $50 \% \mathrm{MCV}$, and $100 \% \mathrm{MCV}$ resistance, respectively, to the posterior occipital area. The system will automatically record and transmit the signal to generate the original image, and indicators of muscle fatigue including median frequency (MF), mean power frequency (MPF), average rectified value (ARV), and root mean square (RMS) will be calculated by Mega Win software for statistical analysis [26].

\section{Secondary outcomes}

The secondary outcomes are measured as described below.

1. Short-form McGill Pain Questionnaire (SF-MPQ): This questionnaire comprises Pain Rating Index (PRI), Present Pain Intensity (PPI), and VAS items. The PRI includes sensory and affective parts with negative, mild, moderate, and severe pain (from 0 to 3 ) representing the degree, while the PPI values from 0 to 5 represent different degrees of pain. A VAS number of 0 represents no pain, and 10 represents unbearable pain [27].

2. Northwick Park Neck Pain Questionnaire (NPQ): The NPQ has been commonly used in clinical research and has shown high validity and practicability for neck pain assessment [28]. It consists of nine items involving the degree and duration of pain, numbness of upper extremities, sleep, and social activities as well as other quality of life characteristics. The total score is 100, and high scores mean severe disability from neck pain.

3. Range of Motion (ROM) of the neck: This reliable and valid tool, consisting of two gravity goniometers and a compass goniometer, is used to assess the degree of cervical spine movement 


\begin{tabular}{|c|c|c|c|c|c|c|c|c|}
\hline & \multicolumn{8}{|c|}{ STUDY PERIOD } \\
\hline & \multirow{2}{*}{$\begin{array}{c}\text { Enrollment } \\
-1 \\
\end{array}$} & \multirow{2}{*}{$\begin{array}{c}\text { Allocation } \\
0\end{array}$} & \multicolumn{4}{|c|}{ Post-allocation } & \multicolumn{2}{|c|}{ Follow-up } \\
\hline $\begin{array}{c}\text { TIMEPOINT* } \\
\text { (week) }\end{array}$ & & & 1 & 2 & 3 & 4 & 8 & 16 \\
\hline \multicolumn{9}{|l|}{ ENROLLMENT: } \\
\hline Eligibility screen & $x$ & & & & & & & \\
\hline Informed consent & $x$ & & & & & & & \\
\hline Medical history & $x$ & & & & & & & \\
\hline Allocation & & $x$ & & & & & & \\
\hline \multicolumn{9}{|l|}{ INTERVENTIONS: } \\
\hline \multicolumn{9}{|l|}{ Jin-acupuncture } \\
\hline \multicolumn{9}{|l|}{ Pseudo acupuncture } \\
\hline \multicolumn{9}{|l|}{ ASSESSMENTS: } \\
\hline PD & $x$ & & & & & $x$ & $x$ & $x$ \\
\hline PR & $x$ & & & & & $x$ & $x$ & $x$ \\
\hline Parameters of SEMG & $x$ & & & & & $x$ & $x$ & $x$ \\
\hline SF-MPQ & $x$ & & & & & $x$ & $x$ & $x$ \\
\hline NPQ & $x$ & & & & & $x$ & $x$ & $x$ \\
\hline ROM & $x$ & & & & & $x$ & $x$ & $x$ \\
\hline SF-36 & $x$ & & & & & $x$ & $x$ & $x$ \\
\hline SAS & $x$ & & & & & $x$ & $x$ & $x$ \\
\hline SDS & $x$ & & & & & $x$ & $x$ & $x$ \\
\hline Adverse events & & & $x$ & $x$ & $x$ & $x$ & & \\
\hline
\end{tabular}

Fig. 2 SPIRIT figure adopts PD and PR of PainVision analyzer, parameters of surface Electromyography (SEMG), short-form McGill Pain Questionnaire (SF-MPQ), range of motion (ROM) of the neck, Northwick Park Neck Pain Questionnaire (NPQ), Self-Rating Anxiety Scale (SAS), SelfRating Depression Scale (SDS) for enrollment, interventions, and assessments

involving the cervical movement distance of bilateral rotation and flexion without undergoing pain [29]. This measurement is performed by a specific senior researcher, and the mean values will be recorded to facilitate the analysis.

4. Short-form 36-item Health Survey (SF-36): The health-related quality of life and correlation factors including physical function and daily functioning are evaluated by a 5-point scale [30].

5. Self-Rating Anxiety Scale (SAS) and Self-Rating Depression Scale (SDS): The SAS is a relatively simple clinical tool to analyze the subjective symptoms of patients. It is applicable to adults with anxiety symptoms and has a wide range of applications. The SDS can fairly directly reflect the subjective feelings of patients with depression and their changes in treatment. It is mainly applicable to adults with depressive symptoms. Compared to the SDS, the SAS can better reflect the subjective feelings of patients with anxiety tendencies [31].

\section{Data collecting and monitoring}

Data will be recorded in electronic case report forms (eCRFs) by specific outcome assessors, and will be established and monitored by the Data Monitoring Committee (DMC) of the First Affiliated Hospital of Yunnan 
Table 1 Treatment details for each group

\begin{tabular}{|c|c|c|}
\hline Group & $\begin{array}{l}\text { Acupoint/location } \\
\text { (affected side) }\end{array}$ & Manipulation \\
\hline $\begin{array}{l}\text { Jin } \\
\text { acupuncture }\end{array}$ & $\begin{array}{l}\text { I. Stress concentration } \\
\text { points } \\
\text { a. In the midpoint of the } \\
\text { connection between BL9 } \\
\text { and BL10 } \\
\text { b. In the midpoint of the } \\
\text { connection between GB20 } \\
\text { and SJ17 } \\
\text { II. Positive reaction points } \\
\text { c. GB8 } \\
\text { d. In the midpoint of } \\
\text { temporomandibular joint } \\
\text { III. Nerve stimulation points } \\
\text { e. The sensitive point of } \\
\text { occipital protuberance of } \\
\text { upper line of posterior } \\
\text { occipital region } \\
\text { f. Sensitive points on the } \\
\text { posterior border of the } \\
\text { upper third of the } \\
\text { sternocleidomastoid } \\
\text { muscle } \\
\text { g. Sensitive spots in } \\
\text { intertubercular sulcus of } \\
\text { the second transverse } \\
\text { process } \\
\text { Matching point: GB34 }\end{array}$ & $\begin{array}{l}\text { a, b, f, g and GB34: } \\
\text { Perpendicular insertion with } \\
1.2 \text { cun } \\
\text { c: Oblique insertion with } 0.8 \\
\text { cun toward the ipsilateral } \\
\text { body } \\
\text { d, e: Oblique insertion with } \\
0.5 \text { cun down } \\
\text { Retaining needles for } 25 \text { min } \\
\text { Lifting-thrusting, twirling, } \\
\text { and rotating for de qi } \\
\text { sensation }\end{array}$ \\
\hline $\begin{array}{l}\text { Pseudo } \\
\text { acupuncture }\end{array}$ & $\begin{array}{l}5 \mathrm{~cm} \text { away from } a, b, c, d, \\
e, f \text { and } g ; 8 \text { points in total }\end{array}$ & $\begin{array}{l}\text { Retaining needles for } 25 \text { min } \\
\text { only, pricking skin with no } \\
\text { manipulation to avoid de qi } \\
\text { sensation }\end{array}$ \\
\hline
\end{tabular}

Number of needle insertions per subject per session will be 8

University of Chinese Medicine. Inspectors will inspect the data, check the study protocol compliance and nonscheduled informed consent documents, and evaluate the conditions of participant recruitment and data quality of this trial. During the evaluation process, the researchers, including acupuncturists and statisticians, will have no eligibility to see the data. Any amendments to this study protocol should be tracked and dated for submittal of a new version to the committee.

According to the data-sharing policy of the Chinese Clinical Trial Registry (CHICTR), data will be transmitted to the official website of Yunnan University of Chinese Medicine (www.ynutcm.edu.cn) within 6 months after this trial is completed. Data will be opened on the condition of publication of the main study findings. In order to protect the participants' confidentiality, all of the external investigators should be asked to sign the agreement. Any adverse events (AEs) or accidents will be observed, reported, and monitored in time until they are resolved. The safety of the trial will be assessed before and after the treatment to avoid AEs, and a strict investigation and relative follow-up monitoring will be performed if they occur.

\section{Adverse events}

Any possible AEs related to acupuncture intervention that occur, including nausea, fainting, subcutaneous hemorrhage, local infection, stuck needles, or needle breakage will be checked and treated. Details of AEs will be recorded in the CRF by the acupuncturist and security administrator. Severe AEs will be reported to the Ethics Committee and DMC, and compensation will be considered if necessary. Patients who are unwilling to persist with the treatment will be removed from the trial.

\section{Statistical analysis}

All of the data will be entered in the CRFs and input into the database by two independent researchers, calculated by means of the SPSS 25.0 software package. Data analysis and comparison will be performed based on the intention-to-treat (ITT) population and the per protocol (PP) population, contributing to the integrity and objectivity of this study. The ITT population consists of all participants who have been randomized and received at least one session of acupuncture after the baseline. PP refers to the patients who have completed the study and did not violate the protocol. A two-sided value of $P<$ 0.05 will be defined as the statistical significance, categorical variables in two groups will be analyzed by chisquared or Fisher's exact test, normally distributed data will be analyzed by Student's $t$ test, and non-normally distributed data by the Mann-Whitney $U$ test. Continuous variables in two groups including primary outcomes (the PainVision and SEMG parameters) and secondary outcomes will be compared using a general linear model with repeated measurement methods at all time points.

\section{Discussion}

$\mathrm{CEH}$ is a subtype of headache derived from a lesion of the cervical spine with accompanying detriment of the surrounding soft tissues [32]. Evidence has indicated that the effective rate and VAS of acupuncture for $\mathrm{CEH}$ is obvious compared to non-steroidal anti-inflammatories and other treatment methods, and few adverse reactions occurred [33-36]. Given that most studies paid more attention to the assessment of the headache, ignoring symptoms and signs of the surrounding soft tissues as well as ROM of the cervical spine [12,37], the quality and reliability of the studies still needs to be improved by carrying out well-designed clinical trials.

From the perspective of jin theory of TCM and modern soft tissue biomechanics $[38,39]$, this study proposes to treat $\mathrm{CEH}$ with specific points: the stress concentration point and positive reaction point. Both of the points are related to trigger pain points and tenderness points, which contain attachment points of occipital-cervical jin to the bones. The points of gather and knot of jin are 
formed due to detriment of greater stress from fascia and tendons to the bones [40]. Thus, loosening anomalous jin can alleviate pain and tension in local soft tissues [41]. In addition, the necessity of therapeutic effect of the nerve stimulation point which is an outlet point of superficial branches of nerves in $\mathrm{CEH}$ is also taken into consideration [42]. Pain is likely to be induced by prolonged flexion of the head and neck and may also be caused by cold stimulation [43].

Studies have shown that superficial punctures under the skin have an analgesic effect on fibromyalgia and other types of chronic pain [44-46]. This trial aims to adopt sham acupuncture, the international general method, as the control condition to evaluate the curative effectiveness of acupuncture based on jin theory. With the use of CEH-related indicator assessment, strict quality control, the guidance of professional experts, and an 8-16 weeks follow-up period, this trial will provide strong evidence for the use of acupuncture treatment for patients with $\mathrm{CEH}$.

\section{Trial status}

This trial (study protocol version pro l, dated 2018-01-16) is ongoing. The recruitment began on 2 April 2018, and the approximate completion date of the trial is 30 April 2020.

\section{Additional file}

Additional file 1: SPIRIT 2013 checklist. (DOC $134 \mathrm{~kb}$ )

Additional file 2: STRICTA checklist. (DOCX $24 \mathrm{~kb}$ )

\section{Abbreviations}

AE: Adverse event; CEH: Cervicogenic headache; CRF: Case report form; ICHD-3: International Classification of Headache Disorders, 3rd edition; MCV: Maximum voluntary contraction; MF: Median frequency; MPF: Mean power frequency; NPQ: Northwick Park Neck Pain Questionnaire; PPI: Present Pain Intensity; PRI: Pain Rating Index; RCT: Randomized controlled trial; ROM: Range of motion (of the neck); SAS: Self-Rating Anxiety Scale; SDS: SelfRating Depression Scale; SEMG: Surface electromyography; SF-MPQ: Shortform McGill Pain Questionnaire: TCM: Traditional Chinese medicine; VAS: Visual analog scale

\section{Acknowledgements}

We would like to acknowledge Professor Shu Wang for advising on this protocol, and also the Department of Tuina, The First Affiliated Hospital of Yunnan University of Chinese Medicine, namely, Yunnan Provincial hospital of Traditional Chinese Medicine.

\section{Authors' contributions}

YKD and TPG are the first co-authors of this article; YKD is responsible for planning the draft and revising the manuscript. YKD, TPG, LX, YX, and SW participated in the design and conception of this trial. CLW is the regulator of the study. YX, LHD, and $M Z$ are responsible for recruiting and treating the patients. ZYZ and GFW are involved in collecting the data. All authors have read and approved the final manuscript and approved the publication of this protocol.

\section{Funding}

This paper is funded by the National Natural Science Foundation (No. 81860884) and by Technology Department-Applied Basic Research Joint
Special Funds of the Yunnan University of Traditional Chinese Medicine (No. 2015FB205(-036)).

\section{Availability of data and materials}

Not applicable.

\section{Ethics approval and consent to participate}

The study is approved by the First Affiliated Hospital of Yunnan University of Chinese Medicine/Yunnan Provincial hospital of Traditional Chinese Medicine (Approval No. 2018-001), registered under number ChiCTR1800015316. The implementation abides by the principles of the Declaration of Helsinki (Version Edinburgh 2000). Participants who meet the inclusion criteria, do not meet the exclusion criteria, and provide written informed consent will be included in this study.

\section{Competing interests}

The authors declare that they have no competing interests.

\section{Author details}

${ }^{1}$ The First Affiliated Hospital of Yunnan University of Chinese Medicine (Yunnan Provincial hospital of Traditional Chinese Medicine), Kunming 650021, China. ${ }^{2}$ Tianjin University of Traditional Chinese Medicine, Tianjin 300193, China. ${ }^{3}$ School of Acupuncture-Tuina and Rehabilitation, Yunnan University of Traditional Chinese Medicine, Kunming 650500, China. ${ }^{4}$ The First Affiliated Hospital of Tianjin University of Traditional Chinese Medicine, Tianjin 300193, China. ${ }^{5}$ The First Affiliated Hospital of Bengbu Medical College, Bengbu 233000, Anhui, China.

Received: 10 October 2018 Accepted: 29 May 2019

Published online: 10 July 2019

References

1. Sjaastad O. Cervicogenic headache: a real headache. Curr Neurol Neurosci Rep. 2011;11:149-55.

2. Cliford G. Pain management in patients with cervicogenic headache. Pain Manage. 2006:21(10):1-12.

3. Headache Classification Committee of the International Headache Society (IHS). The international classification of headache disorders, $3^{\text {rd }}$ edition (beta version). Cephalagia. 2018;38(1):1-211.

4. Headache Classification Subcommittee of the International Headache Society. The international classification of headache disorders, 2nd edition. Cephalalgia. 2004;24:9-160

5. Headache Classification Committee of the International Headache Society (IHS). The international classification of headache disorders, 3rd edition (beta version). Cephalalgia. 2013:33(9):629-808.

6. Sjaastad O, Saunte C, Hovdahl H, et al. "Cervicogenic" headache. An hypothesis. Cephalalgia. 1983:3:249-56.

7. Bogduk N, Govind J. Cervicogenic headache: an assessment of the evidence on clinical diagnosis, invasive tests, and treatment. Lancet Neurol. 2009;8(10):959-68.

8. Zhou L, Hudshakoor Z, Hennessey C, et al. Upper cervical facet joint and spinal rami blocks for the treatment of cervicogenic headache. Headache. 2010;50(4):657-63.

9. Zhou L, Li RC, Zhang DY. Treatment of cervicogenic headache by ultrasoundguided occipital nerve block. J Clin Ultrasound Med. 2012;14(11):762-4.

10. Sluijter ME.Non-thermaL radiofrequency procedures in the treatment spinal pain. Pain in Europe: $2^{\text {nd }}$ annual congress of the European federation of IASP chapters (EFIC). Barcelona: EFIC; 1997. p. 326.

11. Silverman SB. Cervicogenic headache: interventional, anesthetic and ablative treatment. Curr Pain Heatache Rep. 2002;6(4):308-14.

12. Zhang K, Liu Y, Jiang GL. Acupuncture treatment for the cervical headache: a systematic review. Chinese J Pain Med. 2013;19(11):643-7.

13. Dong HT, Tang XZ. Study on the clinical effect of the massage method of micro regulating with vertical cross pressing lying on one side in treating cervicogenic headache. China J Orthop Trauma. 2015;28(8):722-6.

14. Cheng F, Chen Q. Cervicogenic headache treated by dispelling wind and removing meridian obstruction: clinical observation of 76 cases. J Chinese Med. 2013;11(11):1740-1.

15. Wang $Y$, Zhang HY, Wu ZJ, et al. Research progress of applying acupuncture to treat cervicogenic headache. J Sichuan Tradit Chinese Med. 2014;32(8):175-8. 
16. Zhang K, Liu Y, Jiang GL. Acupuncture treatment for cervicogenic headache: a systematic review. Chinese J Pain Med. 2013;19(11):643-7.

17. Sedighi A, Nakhostin Ansari N, Naghdi S. Comparison of acute effects of superficial and deep dry needling into trigger points of suboccipital and upper trapezius muscles in patients with cervicogenic headache. J Bodyw Mov Ther. 2017;21(4):810-4.

18. $X u Y$, Luo L, Lin XM. Study on treatment of cervicogenic headache based on "sinews". J Anhui TCM Coll. 2013;32(6):64-6.

19. Dong YK, Xu L, Sh W. Exploration and analysis of Cervicogenic headache treated by acupuncture based on meridian sinew theory. JCAM. 2019;35(1):76-7.

20. Vincent MB. Headache and neck. Curr Pain Headache Rep. 2011;15:324-31.

21. Sikdar S, Shah JP, Gebreab T, et al. Novel applications of ultrasound technology to visualize and characterize myofascial trigger points and surrounding soft tissue. Arch Phys Med Rehabil. 2009;90:1829-38.

22. Rana MV. Managing and treating headache of cervicogenic origin. Med Clin North Am. 2013;97(2):267-8.

23. Che HL, Hu BC. Triple acupuncture method at cervical Jiaji (EX-B 2) for cervicogenic headache: a randomized controlled trial. Zhongguo Zhen Jiu. 2016;36(1):29-32

24. Ikeno S, Kawamata M. PainVision. Masui The Japanese journal of anesthesiology. 2009;58:1367-72.

25. Maekawa N, Morimoto M, Morimoto M, Uchida T, Umeda T, Koga Y. Can we revaluate pain with PainVision, a device for quantitative analysis of perception and pain? A feasibility study of pain in herpes zoster patients. J Jpn Soc Clin Anesth. 2009;29:824-8.

26. Merletti R. Technology and instrumentation for detection and conditioning of the surface electromyographic signal: state of the art. Clin Biomed. 2009;24(2):122-34.

27. Kachooei AR, Ebrahimzadeh MH, Erfani-Sayyar R, Salehi M, Salimi E, Razi S. Short form-McGill Pain Questionnaire-2 (SF-MPQ-2): a cross-cultural adaptation and validation study of the Persian version in patients with knee osteoarthritis. Arch Bone Jt Surg. 2015;3(1):45-50

28. Cleland JA, Childs JD, Whitman JM. Psychometric properties of the Neck Disability Index and numeric pain rating scale in patients with mechanical neck pain. Arch Phys Med Rehabil. 2008;89:69-74.

29. Youdas JW, Garrett TR, Suman VJ, Bogard CL, Hallman HO, Carey JR. Normal range of motion of the cervical spine: an initial goniometric study. Phys Ther. 1992;72(11):770-80.

30. Lam CL, Tse EY, Gandek B, Fong DY. The SF-36 summary scales were valid, reliable, and equivalent in a Chinese population. J Clin Epidemiol. 2005;58:815-22.

31. Yu X, Cao S, Li J, et al. Applicability research of Self-Rating Anxiety Scale and Self-Rating Depression Scale in termination of pregnancy caused by fetal abnormality. J Nurs Rehabil. 2016;15(8):723-7.

32. Phil P. Cervicogenic headache: an evidenced approach to clinical management. Int J Sports Phys Ther. 2011;6(3):254-66.

33. Zhou XM, Sun $\mathrm{H}$, Chen J. Observation of curative effect of electric acupuncture in occipital area on cervical headache. Shanghai J Acu-Mox. 2008;27(5):30.

34. Chen JB, Li CG. Observations on the efficacy of acupuncture plus massotherapy in treating cervicogenic headache. Shanghai J Acu-Mox. 2016:35(5):517-8.

35. Chu HL, Hu BC. Triple acupuncture method at cervical Jiaji (EX-B 2) for cervicogenic headache: a randomized controlled trial. Chinese Acupunct Moxibustion. 2016;36(1):29-32.

36. Robbins MS, Kuruvilla D, Blumenfeld A, Charleston L IV Sorrell M, Robertson CE, et al. Trigger point injections for headache disorders: expert consensus methodology and narrative review. Headache J Head Face Pain. 2014;54(9):1441-59.

37. Hao CC, Hang XJ, Fan GQ. Suggestion about research for acupuncture treatment of Cervicogenic headache. J Liaoning Univ TCM. 2010;12(5):129-31.

38. Zhang $\mathrm{B}, \mathrm{Li} \mathrm{W}, \mathrm{Chen} \mathrm{W}$. The research progress of the diagnosis and treatment for cervicogenic headache. J Cervicodynia Lumbodynia. 2014;35(1):59-61.

39. Liu NG, Guo CQ. Nature of muscles along regular meridians. Jiangsu J Tradit Chinese Med. 2010;42(8):168-9.

40. Zhu GQ, Fang M, Hong SZ. On the biological mechanic effect of muscle diseases and bone fractures in cervical spondylosis. Beijing J Tradit Chinese Med. 2010;29(1):34

41. Qin XX, Wan Q. Significance of muscle tenderness point needle electromyographic examination in patients with cervicogenic headache. Jiangsu Med J. 2015;41(13):1534-6.

42. Dong $\mathrm{YK}$, Jiang $\mathrm{YW}$, Wang $\mathrm{CL}$, et al. Acupuncture therapy on the focal point of stress and positive reaction point for treating lesions of muscular soft tissues. J Clin Acupunct Moxibustion. 2011;27(3):57-8.
43. Chen J. Clinical evaluation of acupuncture therapy of lingshu jing combined with acumicroprobe loose solution for treating cervicogenic headache. J Clin Acupunct Moxibustion. 2015;31(4):43-5.

44. Gao HY. Overview on the clinical application of nerve cord stimulation therapy. China J Chinese Med. 2012;7:880-1.

45. Linde K, Niemann K, Meissner K. Are sham acupuncture interventions more effective than (other) placebos? A re-analysis of data from the Cochrane review on placebo effects. Forsch Komplementrmed. 2010;17(5):259-64.

46. Meissner K, Fässler M, Rücker $G$, et al. Differential effectiveness of placebo treatments: a systematic review of migraine prophylaxis. JAMA Intern Med. 2013:173(21):1941-51.

\section{Publisher's Note}

Springer Nature remains neutral with regard to jurisdictional claims in published maps and institutional affiliations.
Ready to submit your research? Choose BMC and benefit from:

- fast, convenient online submission

- thorough peer review by experienced researchers in your field

- rapid publication on acceptance

- support for research data, including large and complex data types

- gold Open Access which fosters wider collaboration and increased citations

- maximum visibility for your research: over $100 \mathrm{M}$ website views per year

At BMC, research is always in progress.

Learn more biomedcentral.com/submissions 